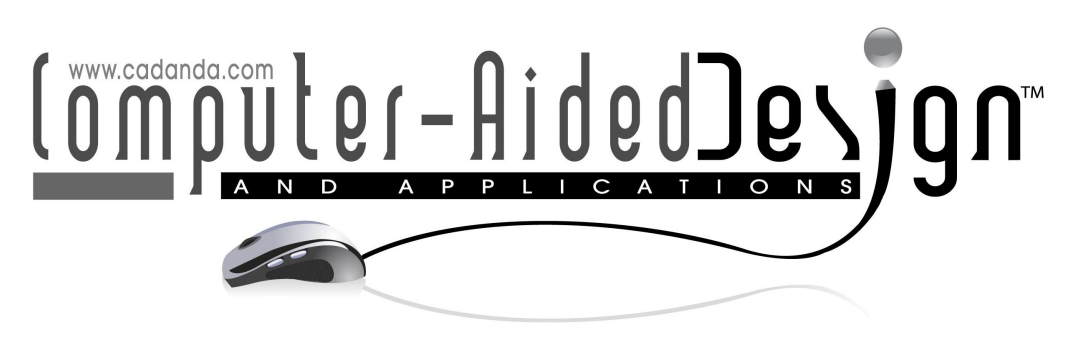

\title{
Graphical Modelling with Computer Extended Descriptive Geometry (CeDG): Description and Comparison with CAD
}

\author{
Manuel Prado-Velasco (D) , Rafael Ortiz Marín (D) , Laura García Ruesgas (D) , M. Gloria del Río Cidoncha (D) \\ University of Seville,
}

Corresponding author: Manuel Prado-Velasco, mpradov@us.es

\begin{abstract}
We present a Computer Extended Descriptive Geometry (CEDG) approach for modelling spatial geometric systems that surpasses several CAD limitations. A first concept proof has shown that the CEDG can be implemented on the dynamic geometry software (DGS) paradigm to generate parametric models based on descriptive geometric techniques. The reliability and performance of the CEDG approach was compared to CAD through two study cases from the sheet metal and mechanisms design fields. The outcomes demonstrate that CEDG is able to compute the design geometrical parameters concurrently with the modelling process and to obtain planar cutouts of 3D surfaces, in situations where CAD systems can not do it. The implementation was performed in Geogebra ${ }^{\odot}$ for CEDG and Solid Edge ${ }^{\odot} 2009$ for CAD, which were selected because of their cutting edge technology. As main conclusion, the CEDG approach is a Descriptive Geometry (DG) - based computer parametric graphical modelling that may complement the CAD technology with accuracy and reliability.
\end{abstract}

Keywords: Descriptive Geometry, Computer Graphic Modelling, Dynamic Geometry Software, Spatial Geometric Analysis, CAD

DOI: https://doi.org/10.14733/cadaps.2021.272-284

\section{INTRODUCTION}

The technical description of spatial geometric systems for industrial design and research requires of accurate procedures to model and communicate geometric and functional specifications of these ones. Technical drawing has evolved from methods based on Monge descriptive geometry [9], adapted and extended to cope with requirements of technical fields [3], towards Computer-Aided Design (CAD) software, which emerged at the middle of 90's. The technical drawing is standardized by the International Standard Organization (ISO), mainly through the ISO 128 . Current CAD paradigm allows the description of mechanical parts and systems directly in the 3D coordinates system. It includes different solid modelling methodologies, based on surfaces geometry and algebraic computational techniques $[1,12]$. 
The parametric definition of geometric elements is an important property of the CAD software, which facilitates the propagation of changes in geometric properties to the final solid part. The evolution of CAD systems include the addition of manufacturing software modules, together with the inclusion of new algorithms to facilitate the analysis of functional behaviour, and the modelling of the relationship between geometric and physical properties [10]. Strategies to link geometric models with their dynamical behaviour include the use of modelling and simulation software tools [8]. In addition, modern CAD tools can be customized through small user computer programs that gain access to the CAD kernel by means of the Application Program Interface (API). A more detailed analysis of the CAD evolution exceeds the scope of this work.

The progressive diffusion of CAD promoved the disuse of descriptive geometry procedures, which are not present in the algebraic computational techniques used in CAD. This issue has been addressed mainly from a teaching perspective, although it has implications on industrial and research areas [13], where CAD presents some relevant limitations. The following brief review of sheet metal engineering and mechanisms' design fields through light on this subject.

The sheet metal engineering field is involved in the calculation of planar cutouts of ducts, polygonal to round transition surfaces, planar and conical hoppers, elbows and other mechanical systems that are manufactured through bending and folding from the flattened state of the metal surface. However, these specialized tasks requires the development of CAD software addons or the incorporation of sheet metal packages, which are limited to a set of supported types of surfaces and patterns an to the assumptions concerning to the folded surfaces reconstruction. The complexity of this field compels to some CAD tools to include several sheet metal modules with different functional scopes.

In opposition to CAD, technical drawing based on descriptive geometry provides complete solutions for nearly any type of sheet metal problem. Any professional with expertise in sheet metal design and descriptive geometry may apply well - known graphical methods [2] and even develop new ones for additional complex situations. Physical entities, like moments of inertia and forces may be obtained during the modelling process to obtain an optimized sheet metal solution.

The design of engineering mechanisms is a mature field where the kinematic analysis and spatial analysis tasks focused to the validation of the coupling chains of limbs need to be performed during the geometric design. Standard CAD tools need to be complemented with external mathematical systems, through iterative procedures, to give a final mechanism's model [16]. However, a software tool based on descriptive geometry procedures complemented with mathematical methods, could solve concurrently both the kinematic and spatial analysis and the geometrical modelling.

Descriptive geometry - based modelling requires the support of computers before it can be applied efficiently. The use of 2D CAD software as drawing board for descriptive geometry modelling does not take advantage of the parametric approach implicit in descriptive geometry, and thus it can not be applied with this goal.

Dynamic Geometry Software (DGS) appears as a computer geometry approach at the beginning of 2000's. It is oriented to the analysis of geometric problems combining algebra and geometry [11]. These software systems link algebraic with geometric descriptions and views. A widely extended DGS system is Geogebra ${ }^{\circledR}$, which adds modules for Computer Algebraic System (CAS), spreadsheet and statistics [5]. DGS systems are considered an evolution of educational geometry programs of 80's, what explains whay DGS research is currently focused on teaching [11]. However, different studies have shown the reliability of DGS in descriptive geometry, parametric geometric constructions and research fields [7, 14].

This work presents the new Computer Extended Descriptive Geometry (CEDG) approach for the modelling of spatial geometric systems. CEDG combines the use of descriptive geometry procedures with parametric modelling, and it will be implemented on the open desktop Geogebra DGS [5]. The specific objectives of the study are: (i) to evaluate the reliability of CEDG as a parametric modelling tool for spatial geometric systems; (ii) to compare the capability and accuracy of CEDG versus CAD (Solid Edge 2019) in two relevant cases from sheet metal and mechanisms' design fields. 


\section{METHODS AND MATERIALS}

CEDG and CAD have the same primary objective, which is the representation of a mechanical system by means of a parametric graphical computational model. However, there are several functional differences that emerge from their underlying principles. Any CAD model is defined by a sequence set of geometric features, built by means of solid generation techniques from plane sketches, surface sweep, boolean operation or surfaces solidification, among others. These features are implemented and organized through a history tree, a direct modeling approach, or a combination of these two main approaches. Any change in a parametric value is propagated in the model according to the type of approach. This is known as regeneration of CAD model.

In contrast, a CEDG model is based on a sequence of algebraic and mathematical entities that can be associated with graphical objects, with a one-to-one relationship. Any entity of the model keeps an algebraic dependence on a set of previous built entities, in such a way that any parametric change in any entity propagates to the subsequent entities, assuring the algebraic consistence of the model. This is the basic principle that supports the integration of descriptive geometry procedures into the CEDG model. Several limitations of CAD tools are expected to be solved under the CEDG approach:

Limitation 1. Descriptive geometry procedures can be integrated in a fundamental form in a CEDG model, in opposition to a CAD model. As a consequence the CEDG approach can apply surface folding processes based on well known techniques for which CAD tools are limited. In addition, the CEDG approach should allow the computation of parameter values that fulfil with geometrical requirements during the process of model building. This technique is similar to the analysis and solution of geometrical systems by means of descriptive geometry techniques, with the advantage of the computational support.

Limitation 2. Kinematic, dynamic and other mathematical equations related to the geometrical properties of the mechanical system can be included in a $\mathrm{CEDG}$ model as mathematical entities, which supports the concurrent computation of geometrical parameters and model building, in opposition to CAD models.

The evaluation of the reliability of $\mathrm{CEDG}$ as a parametric modelling tool for spatial geometric systems is the first specific objective of the study. It includes the ability to modify the geometric properties and projective views of the system through the interactive change of the definition parameters. It will be performed through a concept proof, focused on the modelling of a planar geometrical object in CEDG.

The second specific objective of the study addresses a comparison between CAD and CEDG to evaluate the two expected limitations of CAD aforementioned. A study case from the sheet metal field was designed to test the ability of $\mathrm{CEDG}$ to calculate surface cutouts for which CAD is limited (limitation 1 ). A second study case from the mechanisms engineering field evaluates the capability of CEDG to compute parameter values associated with geometrical and dynamical requirements during the process of model building (limitations 1 and 2).

The following Section defines the concept proof and the study cases and shows their resolution process.

\section{RESULTS AND DISCUSSION}

\subsection{Concept Proof}

The Geogebra modelling mode selected to implement the CEDG approach was deterministic (in opposition to continuous). This mode is related to the underlying computer geometric algebra [6]. Additional details exceed the scope of this work.

Fig. 1 shows the triangular planar form defined by the spatial points $A, B$, and $C$ through their orthogonal views (vertical and horizontal projections), both in the initial position and final position. The later was obtained by means of the parametrization of the rotation angles, which are associated with the graphical sliders $\left(\alpha=38^{\circ}, \beta=108.2^{\circ}\right.$ in the cited figure). The position of the object is controlled through the rotation angles in an interactive way. 


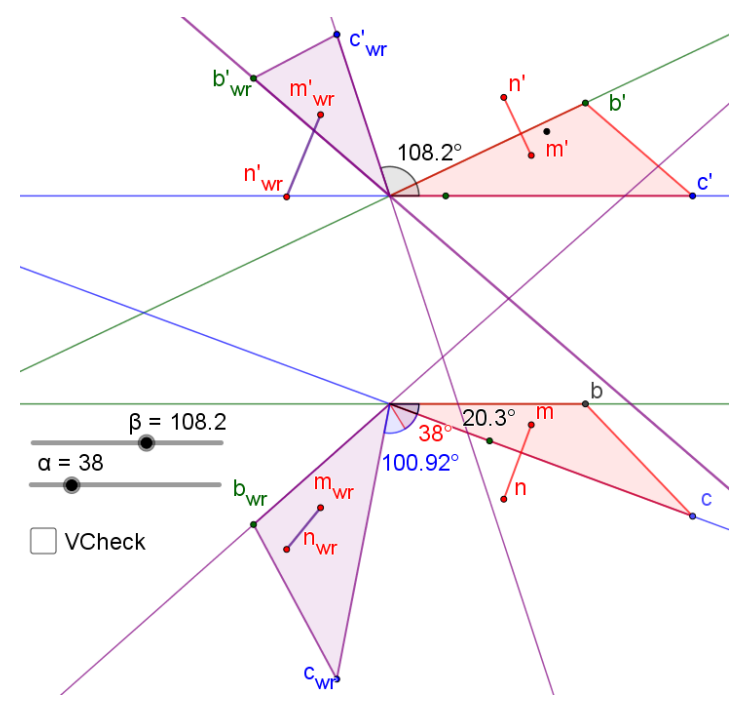

Figure 1: Spatial planar form rotated between two positions by means of the horizontal and vertical angles $\alpha$ and $\beta$ (see text).

The spatial object was completed with a segment MN perpendicular and attached ( $\mathrm{M}$ point) to the planar form. The model was built using descriptive geometry techniques. Graphic entities can be organized into layers. Most of auxiliary graphical constructions have been hidden with the aim of clarity. A logical check box (VCheck, below sliders) that moves the planar form perpendicular to the vertical plane was also implemented and tested with success.

These results confirmed the reliability of the CEDG approach as a parametric modelling tool for spatial geometric systems based on descriptive geometry.

\subsection{Study Cases Definition}

The following list presents a short definition of these ones, which are completed later with the design requirements and accuracy metrics.

1. Sheet metal study case. Calculation of the planar cutout that gives a cylindric hopper bounded by a non-perpendicular planar base and the concave side of a conical cover (see Fig. 2a).

2. Mechanisms design study case. Design and modelling of an horizontal axis jointed door driven by a motor-assisted pulley (see Fig. 2b).

Sheet metal study case. The target cutout must be obtained for a cylindric hopper with a parameterized radius $\mathrm{rCil}$ in the range $1.5-3.5 \mathrm{~m}$, which is compliant with the conical surface dimensions: height $=7.38$ $\mathrm{m}$, diameter $=14.75 \mathrm{~m}$. The cutout will be solved from the folded state of a cylindric surface intersected with the bounding surfaces, according to descriptive geometry procedures [2] for CEDG and using available unfolding commands in CAD. The working surface (null thickness) is defined by the neutral line of the metal sheet as usual. Three procedures will be applied in CEDG:

1. Standard discretization. Intersections between conical and cylindric surfaces are discretized to a finite number of spatial points. Cylinder unfolded state (cutout) is obtained through the substitution of conical arch lengths between directrix points by their chord lengths. 


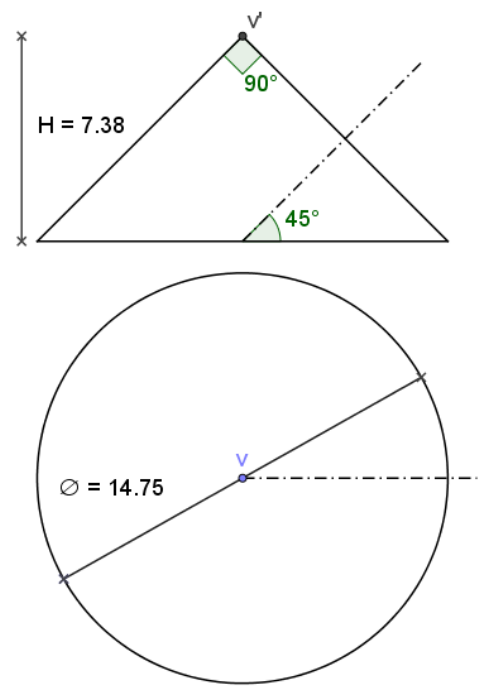

(a) Cylindric hopper given by the axis, planar base and conical cover.

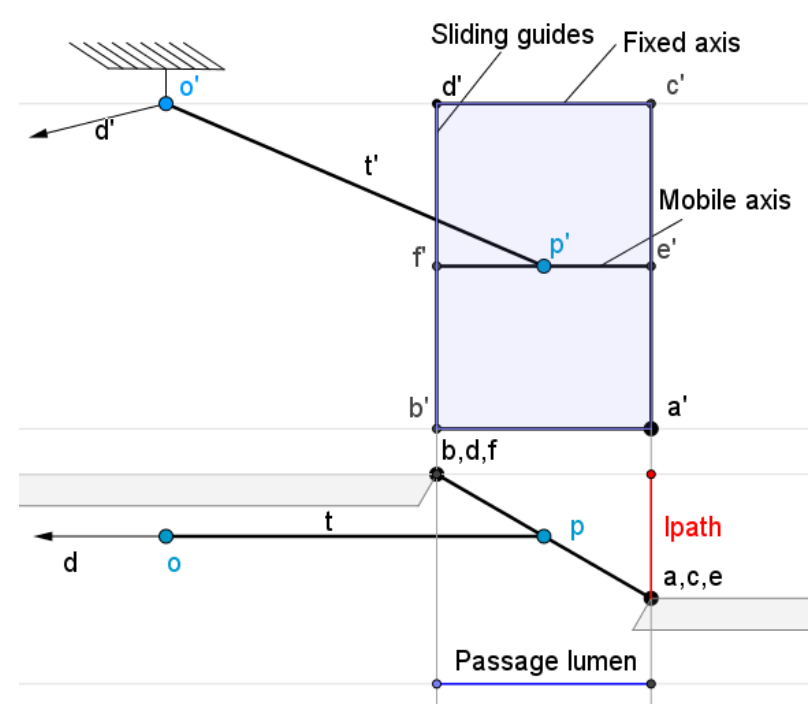

(b) Jointed door defined by two joint thin planar shapes (upper CDFE, bottom ABEF), with mobile axis FE, top fixed axis CD, and vertical sliding guides. Mobile axis is raised through the pulley $\mathrm{O}$ by means of the belt $\mathrm{T}$, connected to the handle $\mathrm{P}$.

Figure 2: Orthographic views of the study cases that are evaluated in the CEDG vs. CAD comparison study.

2. Improved discretization. Similar to standard discretization, but the final cutout is obtained through the true conic arch lengths between cylinder's directrix points.

3. Non-discretized method. Surface intersections are computed combining descriptive geometry procedures with mathematical locus functions. The hopper cutout is calculated in the same way.

The first two methods have been applied successfully during years and they are not limited by software restrictions [2]. The third method requires a computer geometric modelling tool that includes mathematical locus functions, as the Geogebra DGS.

The CEDG cutouts solutions will be compared to the CAD cutout solution, both numerically and methodologically. The accuracy of the cutout will be evaluated by means of the directrix length, $L D$, the connection transformed length (unfolded state of the cylinder - cone intersection), LCint, and the ellipse transformed length (unfolded state of cylinder - plane intersection) $L E$, according to the following equation:

$$
\text { Dif } L_{r e l}=\frac{\left|L-L_{r e f}\right|}{L_{r e f}} \cdot 100 \%,
$$

in which $L$ and $L_{r e f}$ are the evaluated and reference dimension, respectively. The third CEDG method (nondiscretized) is taken as the reference method. The influence of the cylinder radius $\mathrm{rCil}$ and discretization accuracy on the Dif $L$ value will be also analyzed.

Mechanisms design study case. The solution of this study case demands the concurrent calculation of the design parameters and the graphical modelling of the mechanism. The later is composed by two joint planar surfaces that are vertically placed in close position. The horizontal mobile axis (joint) is pulled from handle $P$ by means of the pulley driven by belt $T$. The pulley is placed at point $O$, with the same height than the horizontal fixed axis. The horizontal lower bar BA raises inside vertical sliding guides during the opening process. Door's and pulley's height $\left(H_{d}\right)$ and passage lumen are fixed according to external enclosures as 
$10.30 \mathrm{~m}$ and $6.77 \mathrm{~m}$, respectively. The functional height when the door is fully opened must be $H=10 \mathrm{~m}$. Initial position of the belt $\mathrm{T}$ (closed door) must be parallel to the enclosure, as shown in Fig. $2 \mathrm{~b}$.

Due to the restriction on the position of the belt $\mathrm{T}$, the jointed door will be skewed with respect to the enclosures as presented in the horizontal view of Fig. $2 b$, where the value of lpath dimension (and thus the door's width) needs to be calculated to optimize the operation. The optimization criteria for this design parameter are:

(i) Static motor torque must be minimized.

(ii) Planar shapes must operate with minimal compression strength.

(iii) The value Ipath must be as small as possible to reduce the door's skew position.

The study case solution includes a parametric model of the jointed door that describes the opening - closing operation process. This operation process should be managed through the angle between planar shapes, alfa, which can theoretically vary in the range $0^{\circ}$ (fully open) to $180^{\circ}$ (fully closed). The static forces transmitted to the handle $\mathrm{P}$ at any operation point will be included in the parametric model.

\subsection{Cylindric Hopper in CeDG vs CAD}

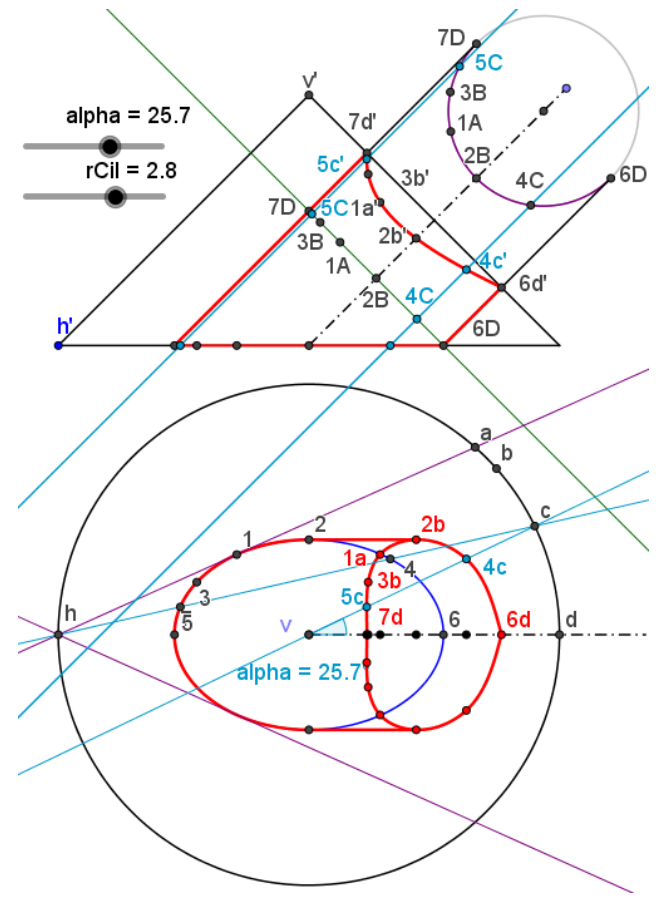

(a) Orthographic views of 3D solution.

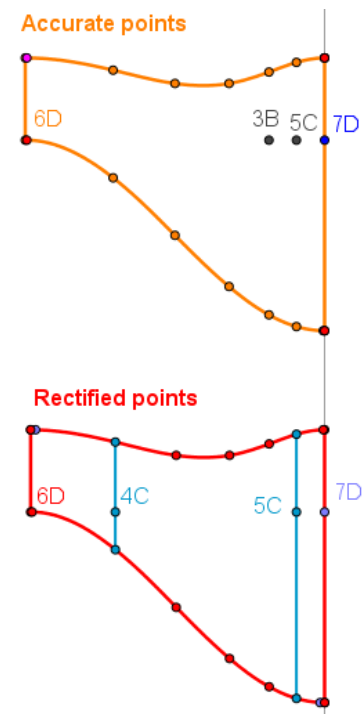

(b) Half-cutouts.

Figure 3: Cylindric hopper 3D solution with $r C i l=2.8 \mathrm{~m}$ for the two first CEDG methods (left) and half-cutouts for the first (bottom-right) and second (upper-right) CEDG methods.

The 3D cylindric hopper solution obtained using any of the first two CEDG procedures is shown in Fig. 3 a for a cylinder radius of $2.8 \mathrm{~m}$, as the $\mathrm{rCil}$ slider shows. The hopper model includes the algebraic entities 
associated with the graphic ones presented in the figure. The base edge is an ellipse given by the intersection of bottom plane with cylinder, whereas the conical cover is a fourth grade spatial curve produced by the intersection of cylinder and cone.

The ellipse is defined by its conic equation, whereas the upper cover edge has been obtained through an interpolation curve with 12 spatial points defined as $1 \mathrm{~A}, 2 \mathrm{~B}, 3 \mathrm{~B}, 4 \mathrm{C}, 5 \mathrm{C}$, plus the symmetric ones, besides $6 \mathrm{D}$ and 7D. The interpolation curve was generated through a cubic spline, without any other condition. The accuracy of the cover edge can be improved incrementing the number of points, and with the use of additional geometric properties. For instance, the continuity of the curve derivative implies that the tangent line at $6 \mathrm{D}$ must be unique, and therefore the cover edge should be perpendicular to the cylinder axis at $6 \mathrm{D}$ in the horizontal view. This property has not been applied in the solution of Fig. 3a, to remark that it is a condition that must be known by the modelling expert under the CEDG approach. However, this imprecision does not affect to their associated cutouts, as is explained below.

The orthographic views of the 3D hopper marks in blue the constructive elements associated with edge points $5 \mathrm{C}$ and $4 \mathrm{C}$. They are generated by a plane that contains a cone generatrix defined by angle alpha (equal to $25.7^{\circ}$ in Fig. 3a). This variable is managed by the alpha slider to move the $C$ point between $B$ and $D$ points at the cone directrix. The accuracy of the cylinder upper cover edge discretization depends on the alpha value.

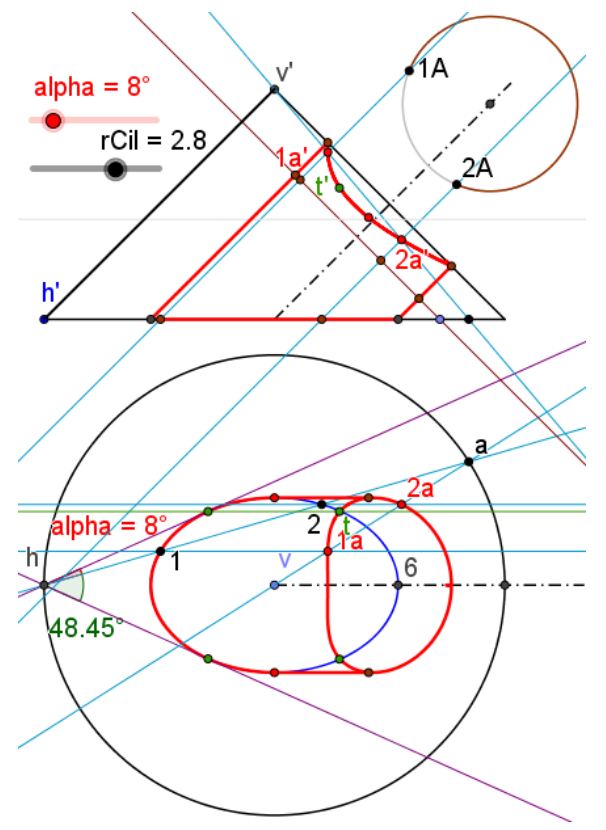

(a) Orthographic views of 3D solution.

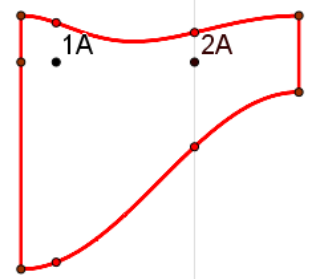

(b) Half-cutout.

Figure 4: Cylindric hopper 3D solution with $r C i l=2.8$ for the third CEDG method (reference) (left) and its halfcutout (right).

Fig. 3b shows the half-cutouts of the cylindric hopper obtained through the folded state, according to the first two CEDG procedures. We have taken advantage of the symmetry of the unfold state with respect to the cylindric axis. The cutout edges are calculated through cubic spline interpolation with points at the unfold state. Therefore, their accuracy do not depend on the interpolation applied to the folded hopper edge (Fig. 3a).

The discretization induces a shorter directrix length, $L D$ in the first CEDG method with respect to the true perimeter length obtained with the second CEDG method. The LD differences are shown in Table 1. 
Fig. 4a shows the hopper solution obtained with the third CEDG method for $r C i l=2.8 \mathrm{~m}$. An auxiliary plane that contains points 1 and 2 from cylinder bottom edge and point $A$ from cone directrix was used to calculate points $1 \mathrm{~A}$ and $2 \mathrm{~A}$ at upper cover edge. This plane is controlled with the alpha angle (with respect to the limit plane L1, horizontal view) in the horizontal view of Fig. 4a. The cylinder - cone intersection was obtained through the following equations (2):

$$
\begin{aligned}
& \text { Cedge } 1 A=\operatorname{locus}(Q, \text { alpha }) \\
& \text { Cedge } 2 A=\operatorname{locus}(S, \text { alpha }),
\end{aligned}
$$

in which $Q$ and $S$ are the names of the points (model entities) with labels $1 A$ and $2 A$, respectively. The locus function generates the intersection curve when the parameter alpha moves along the whole slider range: $0^{\circ}$ (auxiliary plane equal to L1 limit plane) - $48^{\circ}$ (auxiliary plane equal to L2 limit plane). A comparison between CEDG models of Fig. 3a and Fig. 4a shows that the third CEDG method corrects the inaccuracy of the 3D cover edge at $6 \mathrm{D}$ point commented previously. The half-cutout of the cylindric hopper generated according to the third CEDG method is shown in Fig. 4b. The unfold border curves are obtained using the equations (2), where $Q$ and $S$ points are substituted by points pertaining to the unfold edges.

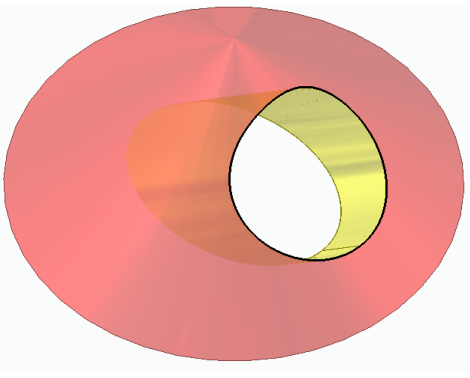

(a) Rendered solid model.

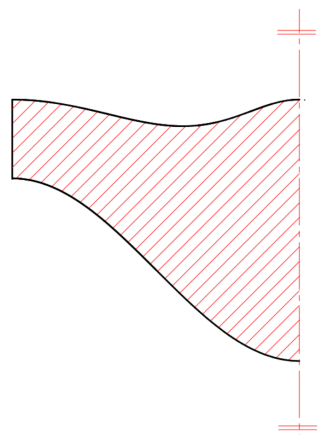

(b) Half-cutout.

Figure 5: Cylindric hopper 3D shell (see text) with $r C i l=2.8$ (left) and its half-cutout (right) computed using Solid Edge 2019.

Solid Edge 2019 was not able to unfold the spatial cylindric hopper surface limited by the conical connection. We created a solid cylinder limited by planar and conical surfaces, which was emptied out to give a cylindric shell with minimal width $(1 \mathrm{~mm})$ and open bottom and top connection faces, in order to overpass that limitation. In addition, a small piece of cylindric shell material was removed along the smallest cylindric generatrix, before the hopper could be unfolded to obtain the cutout solution presented in Fig. 5b. The influence of this removal $(\approx 1 \mathrm{~mm}$ of directrix length) on the cutout dimensions can be neglected due to the hopper directrix dimension $(\approx 20 \mathrm{~m})$. The cylindric shell generated with Solid Edge following the cited procedure is presented in figure 5 a. The external conical surface (red) was intersected with the solid cylinder to define the upper border of the cylindric hopper.

The relative differences of the cutout lengths as a function of the cylinder radius, comparing the CEDG approach to CAD, are shown in Table 1 . The influence of the discretization on CEDG methods 1 and 2 is managed by means of the angle alpha (see Fig. 3a), with values $25.7^{\circ}$ and $35^{\circ}$.

As shown, the accuracy of the CAD cutout is very close to the accuracy of CEDG 2. These two modelling techniques obtain a value of $L D$ (directrix length) with null error, whereas border lengths ( $L C$ int and $L E$ ) had errors about $0.5 \%$. This results suggest that CAD uses a discretization procedure similar to that used in $\mathrm{CEDG} 2$, in agreement with usual computational geometric algebra interpolation methods of CAD tools 
Table 1: Half-lengths $(\mathrm{m})$ and relative differences (\%) of the discretized CEDG methods 1 and 2, besides CAD, with respect to the reference $C E D G$ method 3 .

Reference CeDG 1 CeDG 2 CAD

\begin{tabular}{lcccc}
\hline$r C i l=2.8 \mathrm{~m}$ & \multicolumn{4}{l}{} \\
\hline LD & 8.796 & $8.639(1.78) / 8.513(3.22)$ & $8.796(0) / 8.796(0)$ & $8.796(0)$ \\
LCint & 8.938 & $8.832(1.19) / 8.707(2.58)$ & $8.987(0.55) / 8.986(0.54)$ & $8.988(0.55)$ \\
$L E$ & 10.647 & $10.563(0.79) / 10.458(1.78)$ & $10.696(0.46) / 10.700(0.50)$ & $10.696(0.46)$ \\
\hline$r C i l=3.5 \mathrm{~m}$ & \multicolumn{5}{l}{} \\
\hline$L D$ & 11.0 & $10.78(2.0)$ & $11.0(0)$ & $11.0(0)$ \\
$L C$ int & 11.357 & $11.210(1.29)$ & $11.418(0.54)$ & $11.423(0.58)$ \\
$L E$ & 13.305 & $13.200(0.79)$ & $13.372(0.50)$ & $13.371(0.50)$ \\
\hline
\end{tabular}

The lengths values obtained for CEDG methods 1 and 2 are given for two alpha discretization values $\left(25.7^{\circ} / 35^{\circ}\right)$.

[15]. In addition, the length errors increased with the radius of the cylinder in CAD and CEDG 1 and 2, when the discretization accuracy is kept.

The influence of the discretization accuracy was studied by modifying the alpha value from $25.7^{\circ}$ to $35^{\circ}$ in CEDG 1 and 2, which is associated with the movement of $C$ point towards $B$ point in the parametric model of Fig. 3a (horizontal view). As shown in Table 1, length errors grow with alpha, in agreement with the decrease of discretization accuracy.

The addition of new connection borders (lateral circular holes) in the cylindric hopper gives a 3D surface that could not be unfolded by Solid Edge 2019. Similar problems were reached when we tried to unfold transition surfaces between polygonal and conic holes in CAD. In contrast, the cylindric hopper was successfully unfolded with lateral circular holes and with all modifications that were evaluated. This study case demonstrates that the CEDG is a reliable and accurate parametric modeling approach in sheet metal field. It surpasses the limitations of modern CAD systems with regard to the unfolding of spatial surfaces.

\subsection{Jointed Door System in CeDG vs CAD}

The design of a jointed door that fulfils the requirements stated in the Methods Section compels to build a parametric model that facilitates the geometric and functional analysis. The design variables are included as parameters. The orthographic vertical and horizontal views of this model, built under the CEDG approach, are presented in Fig. 6.

The passage lumen is a fixed length $(1.44 \mathrm{~m})$ labeled in the horizontal view. The angle between door planar shapes, alfa, is related to the functional height, labeled in the vertical view (height). We have taken alfa and lext (lext = Ipath $+1.44 \mathrm{~m}$, where Ipath is the path length to be minimized) as model parameters controlled through sliders, as shown in Fig. 6. The parameter Ipath is a design parameter to be calculated, whereas alfa controls the opening-closing process. Initial range for alfa was $0-180^{\circ}$. The range for Ipath was not known initially. The jointed door model presented in the figure 6 assigns lpath $=3.91 \mathrm{~m}$ (lext $=5.35 \mathrm{~m})$, and alfa $=64.51^{\circ}$ (Height $H=4.78 \mathrm{~m}$ ). The thickness of the planar shapes is not included since it simplifies the graphical modelling, but it has considered in the functional design (equations (3) below), which is also included in the CEDG model.

The pulley driven by belt $\mathrm{T}$ exerts a tensile force $\mathrm{Fa}$ on the handle $\mathrm{P}$ that raises the door. Model of Fig. 6 shows the handle position both at the initial closed state (point $P$ through projections $\left(p^{\prime}, p\right)$ ) and at the position alfa $=64.51^{\circ}\left(\mathrm{P}_{\mathrm{p}}\right.$ through projections $\left.\left(\mathrm{p}_{p}^{\prime}, \mathrm{p}_{p}\right)\right)$. For a generic position of the jointed door, the belt 


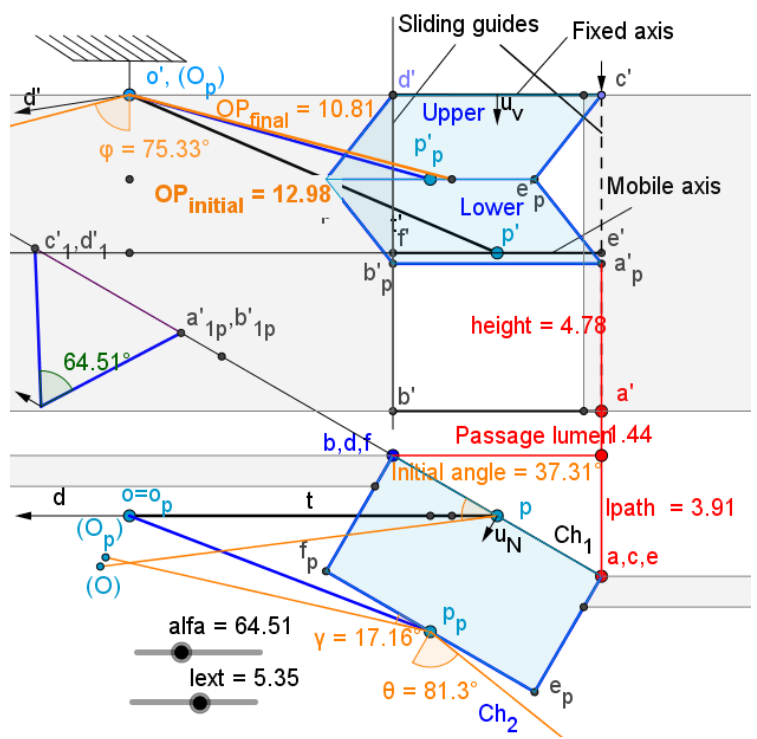

Figure 6: Orthographic views of the jointed door created under the CEDG approach.

T have angles $\gamma, \phi$ and $\theta$ with the horizontal mobile axis, the vertical direction (referred as $u_{v}$ ) and the normal direction (perpendicular to the mobile axis and inside an horizontal plane, referred as $u_{N}$ ), respectively. Any of these angles can be graphically calculated by folding the plane that contains it on a plane parallel to a projection view.

Their values for the generic system position of Fig. 6 are $\gamma=17.16^{\circ}, \phi=75.33^{\circ}$ and $\theta=81.3^{\circ}$, as indicated. Belt $T$ lengths from pulley to handle are also given for the $P(12.98 \mathrm{~m})$ and $P_{p}(10.81 \mathrm{~m})$ positions. The model can be programmed to open/close the jointed door with a desired speed through the alfa operation slider. This feature facilitates the visual inspection of the system behaviour (including the values of geometric and functional variables).

Projected components of $\mathrm{Fa}$ are $\mathrm{Fa}_{v}$ (vertical direction), $\mathrm{Fa}_{n}$ (normal direction) and $\mathrm{Fa}_{t}$ (tangential direction). Static forces involved on the jointed door movement process are normalized with respect to the characteristic weight of the jointed door, which is linearly related to the characteristic width, widtho, to facilitate the numerical analysis of forces. We assign width ${ }_{\circ}=6.9 \mathrm{~m}\left(\operatorname{lext}_{\mathrm{o}}=2.80 \mathrm{~m}\right)$, and then $\mathrm{Fa}_{v}=$ width $/$ width $_{\mathrm{o}}$, and $\mathrm{Fa}=\mathrm{Fa}_{v} / \cos \phi$. The remaining components can be written as:

$$
\begin{aligned}
& \mathrm{Fa}_{n}=\mathrm{Fa} \cdot u_{n}=\mathrm{Fa} \cos \theta \\
& \mathrm{Fa}_{t}=\mathrm{Fa} \cdot u_{t}=\mathrm{Fa} \cos \gamma,
\end{aligned}
$$

where $u_{n}$ and $u_{t}$ are unitary normal and tangential vectors.

Door weight must be equilibrated with the vertical projection of the applied force, what explains the definition of $\mathrm{Fa}_{v}$. We operate with static forces according to the requirements. The minimization of compression loads in door's planar shapes can be achieved if $\mathrm{Fa}_{n} \geq 0$.

The normal force $\mathrm{Fa}_{n}$ is reduced with door's width (an thus with lext) when alfa is constant. This behaviour is shown in Fig. 7a (functional plots obtained through the CEDG model) for alfa $=0^{\circ}$ (fully open). The solution of $\mathrm{Fa}_{n}$ (lext) $=0$ gives lext $=4.658 \mathrm{~m}$, which is the minimal lext value for alfa $=0^{\circ}$ that guarantees $\mathrm{Fa}_{n} \geq 0$.

The value of lext that assures $\mathrm{Fa}_{n} \geq 0$ must be computed for the lowest operative alfa value (alfa $a_{\min }=$ 


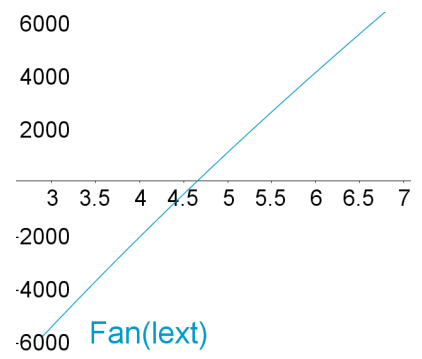

(a) Normal force $\mathrm{Fa}_{n}$ (p.u.) at alfa $=0^{\circ}$ vs. lext $(\mathrm{m})$.

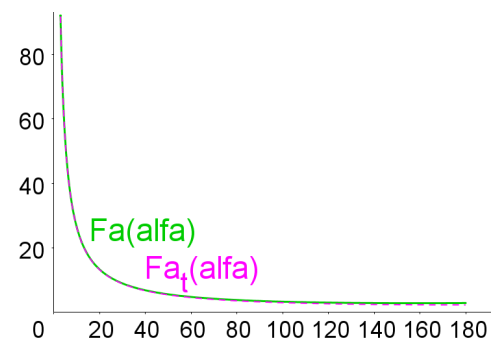

(b) Forces $\mathrm{Fa}$ and $\mathrm{Fa}_{t}$ (p.u.) vs. $\left.\operatorname{alfa}^{\circ}\right)$.

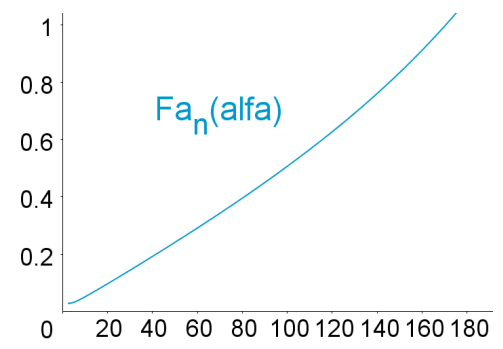

(c) Force $\mathrm{Fa}_{n}$ (p.u.) vs. alfa $\left({ }^{\circ}\right)$.

Figure 7: Normalized $\mathrm{Fa}_{n}$ force as a function of the lext $(\mathrm{m})$ design parameter for alfa $=0^{\circ}$ (a); $\mathrm{Fa}$ and $\mathrm{Fa}_{t}(\mathrm{~b})$ and $\mathrm{Fa}_{n}$ (c) as a function of alfa $\left(2.81^{\circ}-180^{\circ}\right)$ for lext $=4.66 \mathrm{~m}$.

$\left.2.81^{\circ}\right)$, which occurs when the door is open to provide the requested height $\left(H\left(\right.\right.$ alfa $\left.\left._{\min }\right)=10 \mathrm{~m}\right)$. The solution of $\mathrm{Fa}_{n}$ (lext) $=0$ for alfa $=2.81^{\circ}$ is lext $=4.659 \approx 4.66 \mathrm{~m}$.

Design requirements demand also a minimal static motor torque. As the motor torque (and thus $\mathrm{Fa}$ ) is maximal with alfa $=2.81^{\circ}$ (fully open door), and $\mathrm{Fa}_{t}$ does not depend on lext, it is deduced that $\mathrm{Fa}$ is minimal when $\mathrm{Fa}_{n}=0$. We conclude that optimal lext $=4.66 \mathrm{~m}$, and thus optimal Ipath $=4.66-1.44=3.22 \mathrm{~m}$ (optimal door's width $=7.50 \mathrm{~m}$ ). This is the required solution of Ipath design parameter.

The ability of CEDG approach to perform functional analysis concurrently with the graphical modelling is remarked if we examine the static force's components during the operation cycle (Fig. 7b and 7c). Although the designed mechanism fulfils with the optimization criteria, the static force Fa applied at the door handle in full open position is around 83 times the door's weight, what is very inefficient. However, it is possible to limit Fa below 10 if the full open position occurs for alfa $a_{\min }=27^{\circ}$ (height $=7.86 \mathrm{~m}$ ). The minimal Fa increases to 0.12 in this case, what is a better solution to minimize compression efforts. This alternative solution could be implemented through the inclusion of $H_{d}$ (defined as $10.30 \mathrm{~m}$ ) as a new design parameter in the CEDG model.

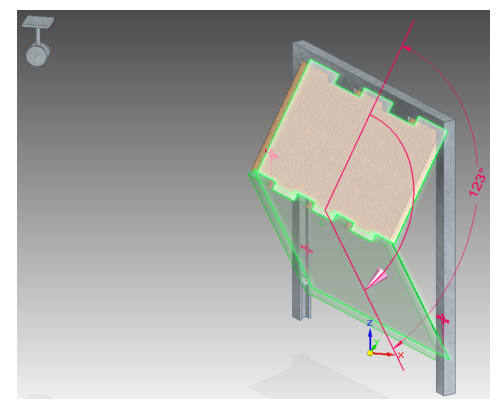

Figure 8: Rendered CAD model of the designed jointed door

It was not possible to implement this type concurrent functional - graphical modelling with Solid Edge 2019. However, Solid Edge allowed an efficient 3D parametric modelling of the mechanism once the design parameters were known. This model is presented in Fig. 8 for the design value of the door's width $(7.5 \mathrm{~m})$, and the operation position alfa $=123^{\circ}$.

This study case demonstrates that CEDG is a reliable approach to design and model mechanisms, thanks to the descriptive geometry foundation, and the capability to implement functional relationships associated 
with the design process (e.g. Fa ${ }_{n}$ (lext, alfa)). The CAD technique (Solid Edge 2019) was not able to compute the design values. The dynamic modification of the parametric model in CEDG was also a key feature to visualize the different design solutions and operation cycles.

\section{CONCLUSIONS}

This work presents a new parametric modelling approach of geometric spatial system founded on descriptive geometry, CEDG, which solves several limitations of CAD systems.

First, conclusions of the concept proof confirm that the Dynamic Geometry Software (DGS) paradigm is able to support the CEDG approach. Secondly, the two study cases from the sheet metal and mechanisms design fields were implemented on Geogebra - based CEDG and Solid Edge 2019 CAD. The outcomes show that $\mathrm{CEDG}$ is able to calculate accurately the required design parameters during the graphical modelling process, and to unfold different spatial cylindric hopper systems. In contrast, the CAD tool presented relevant lacks that impeded to solve both study cases.

The software selected to implement the study cases, Geogebra for CEDG and Solid Edge 2019 for CAD, represents the cutting edge in their respective fields. Although our conclusions have been achieved for this software, the technologies underlying other well-known CAD tools are similar, and thus equivalent lacks are expected in them. Preliminary research has been performed with $\mathrm{CATI}^{\odot}$ and AutoCAD $^{\odot}$, although additional work is needed to generalize the results.

The analysis of differences between the geometry algebraic computational methods that support CAD software [15] and DGS software [6,4] exceeds the scope of this work. However, preliminary analysis confirm the possibility to add curves and surfaces interpolation techniques to DGS that are present in CAD, like B-splines.

Manuel Prado-Velasco, http://orcid.org/0000-0003-2969-0423

Rafael Ortiz Marín, http://orcid.org/0000-0003-0286-5369

Laura García Ruesgas, http://orcid.org/0000-0002-1856-6550

María Gloria Del Río Cidoncha, http://orcid.org/0000-0002-6074-6838

\section{REFERENCES}

[1] de Berg, M.; van Kreveld, M.: Computational Geometry. Algorithms and Applications. Springer, 2000.

[2] Díaz, E.D.: Tratado de trazados y desarrollos de Calderería [Treatise of drawing and development in sheet metal]. Marcombo, Barcelona, Spain, 2010. ISBN 9788426715579.

[3] Graves, O.: Orthographic projection: the elementary principles of orthographic projection, with their applications to technical drawing. Eschenbach brothers, University of Chicago, 1912.

[4] Hildenbrand, D.; Oldenburg, R.: Geometric algebra: A foundation of elementary geometry with possible applications in computer algebra based dynamic geometry systems. The Electronic Journal of Mathematics and Technology, 1(1), 1-19, 2007.

[5] Hohenwarter, M.; Fuchs, K.: Combination of dynamic geometry, algebra and calculus in the software system GeoGebra, 128-133. Sarvari, Cs. Hrsg., Pecs, Hungary, 2005.

[6] Kortenkamp, U.: Foundations of Dynamic Geometry. Thesis, Swiss Federal Institute of Technology, Zurich, 1999.

[7] Kovács, Z.; Recio, T.; Richard, P.R.; Vélez, M.P.: Geogebra automated reasoning tools: A tutorial with examples. In ICTMT, 400 - 404, 2013.

[8] Mejía-Gutiérrez, R.; Carvajal-Arango, R.: Design verification through virtual prototyping techniques based on systems engineering. Research in Engineering Design, 28(4), 477-494, 2017. 
[9] Monge, G.: An elementary treatise on descriptive geometry, with a theory of shadows and of perspective. Oxford University, London, 1851.

[10] Palmer, R.S.; Shapiro, V.: Chain models of physical behavior for engineering analysis and design. Research in Engineering Design, 5(3), 161-184, 1993.

[11] Preiner, J.: Dynamic Mathematics Software to Mathematics Teachers : the Case of GeoGebra. Thesis, University of Salzburg, 2008.

[12] Schneider, P.J.; Eberly, D.H.: Geometric Tools for Computer Graphics. Morgan Kaufmann Publishers, 2002.

[13] Stachel, H.: The status of todays descriptive geometry related education (cad/cg/dg) in europe. Journal of Graphic Science of Japan, 41, 15-20, 2007.

[14] Surynková, P.: Central and parallel projections of regular surfaces: Geometric constructions using 3d modeling software. In ICTMT, 169-176, 2013.

[15] Wintz, J.: Algebraic Methods for Geometric Modeling. Thesis, University Nice Sophia-Antipolis, Nice, France, 2008.

[16] Yu, W.; Wang, H.; Chen, G.: Design and kinematic analysis of a 3-translational-dof spatial parallel mechanism based on polyhedra. Mechanism and Machine Theory, 121, 92-115, 2018. 